\title{
News
} \& Update

\section{Emerging Leaders Alliance; TMS ICME Implementation Study Update}

\section{TMS Members Develop Their Leadership Potential as ELA Participants}

TMS was proud to send five promising members to the 2012 Emerging Leaders Alliance (ELA) Capstone Program, a leadership training opportunity for scientists and engineers working in management, technical, and academic positions. Attending the program were: Paul Ohodnicki, National Energy Technology Laboratory; Nima Rahbar, Worcester Polytechnic Institute; Pello Uranga, CEIT; Ed Herderick, Edison Welding Institute; and Dwayne Shirley, Texas Instruments.

ELA is a multi-society initiative that provides an interdisciplinary community of learning for engineering and scientific professionals. The intent of ELA is to prepare future engineering leaders for the challenges they will face in advancing their organizations within complex business and social environments. It accomplishes this by providing a comprehensive leadership development experience that culminates with the three-day Capstone Program. TMS and the TMS Foundation were Gold Level Sponsors for the 2012 ELA Capstone Program, which took place September 11-13 in Reston, Virginia.

Admission to the ELA is competitive. Applicants must be members of TMS, typically ages $25-40$, and be individuals with rising or current technical staff leader positions, potential for upper-level management or professors with aspirations for university leadership roles. For additional information on ELA or how to apply for the 2013 program, visit the website at emergingleadersalliance.org or contact Deborah Price, TMS awards

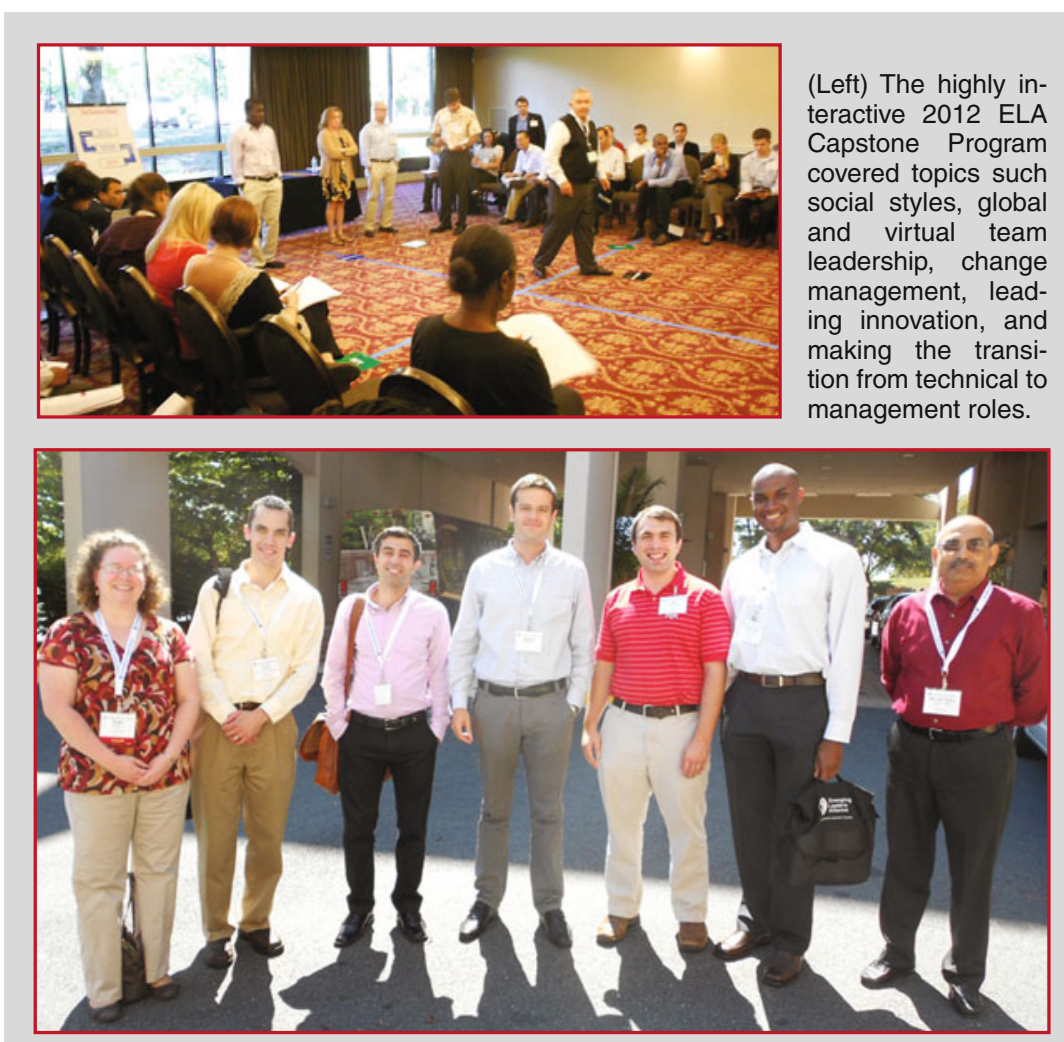

Attending the 2012 ELA Capstone Program were (left to right): Deborah Price, Paul Ohodnicki, Nima Rahbar, Pello Uranga, Ed Herderick, Dwayne Shirley, and Brajendra Mishra, 2006 TMS president and 2011 AIME president.

and recognition specialist, at price@ tms.org.

\section{TMS ICME Implementation Study Teams Begin Work}

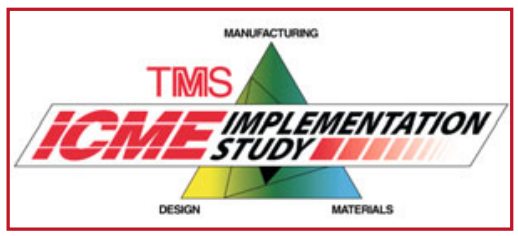

TMS announced in September that it has formed the teams that will study and identify key steps needed to extend and accelerate implementation of the emerging discipline of integrated computational materials engineering (ICME). TMS, which is leading the project on behalf of the U.S. Department of Defense, the U.S. Department of Energy, and the National Science Foundation, has recruited a cross section of more than 35 scientists, engineers, and technical experts to serve on a total of four ICME implementation study teams. Three of these teams will focus on specific industrial sectors - aerospace, automotive, and maritime-while a fourth team will study the "cross-cutting" issues across these sectors.

"We are pleased to be able to assemble and convene such a broad and rich talent base for this important project," said George Spanos, TMS technical director and the study's project leader. "Engaging experts from industry, academia, and government will not only provide a much more comprehensive and detailed approach than would be possible by any single organization; it will also facilitate the implementation of ICME across the entire materials design and development cycle-from discovery to deployment."

Providing leadership for each of the four ICME implementation study teams convened by TMS are:

- Tresa Pollock, Aerospace Team leader, is Alcoa Professor of Materials and chair, Materials Department, University of California at Santa Barbara. 
- John Allison, Automotive Team leader, is professor, Materials Science and Engineering, University of Michigan at Ann Arbor.

- Johnnie Deloach, Maritime Team leader, is manager, Welding, Processing and Non-destructive Evaluation Branch, Naval Surface Warfare Center; technology transition detailee, Office of Naval Research.

- Brad Cowles, Cross-Cutting Team leader, is with Cowles Consulting LLC and senior fellow and discipline lead (retired) for Materials and Processes, Pratt \& Whitney.

"This new study will determine frameworks and pathways needed to implement ICME more rapidly and on a much broader scale, within the automotive, aerospace, and maritime industries, and will generate ideas and offer guidance that can aid integrated product development teams, ICME practitioners, and research and engineering groups seeking to implement ICME in these industries," said Spanos. "The intent is that the final report will, in essence, serve as a 'field manual' toward such ICME implementation."

\section{TMS Welcomes New Members}

At its September 2012 meeting, the TMS Board of Directors approved a slate of new members representing an array of materials science and engineering disciplines. Please join us in congratulating the following individuals as they begin to explore the many personal and professional growth opportunities offered to them as full members of TMS:

Adachi, Yoshitaka, Kagoshima University, Japan

AlGrafi, Mubarak W., Taibah University, Saudi Arabia

Anderson, Ken R., Bechtel Marine Propulsion Corp., United States

Anwar, Shahriar, Arizona State University, United States

Avishai, Nanthawan, Case Western Reserve University, United States
Barakat, Mohamed A., Central Metallurgical Research \& Development Institute, Egypt

Barton, Nathan, Lawrence Livermore National Laboratory, United States

Ben Salah, Nihad, Heroux-Devtek, Canada

Bluhm, Madison M., Total Petrochemicals and Refining USA, United States

Boller, Elodie, ESRF, France

Bouman, Charles A., Purdue University, United States

Buffiere, Jean-Yves, INSA, France

Case, Eldon Darrel, Michigan State University, United States

Cashwell, William J., United States

Chupin, Sylvain, CEA, France

Ciccotti, Richard G., Magnesium Elektron North American Inc., United States

Colligan, Kevin J., Nova Tech Engineering, United States

Crawford, Grant, South Dakota School of Mines \& Technology, United States

Croll, Alexander B., General Motors, United States

Curtis-Rouse, Mike, Harwell Imaging Partnership, Great Britain

Dionne, Jean-Francois, The Swatch Group R\&D Division Asulab, Switzerland

Dogan, Gunay, National Institute of Standards and Technology, United States

Downs, James J., Rhenium Alloys Inc., United States

Draney, Cameron K., United States

Drosback, Meredith, United States

Drummy, Larry, UES Inc., United States

Ercius, Peter A., National Center for Electron Microscopy, United States
Fahey, Kevin P., Xradia, United States

Ferro, Patrick D., Gonzaga University, United States

Fife, Julie L., Paul Scherrer Institute, Switzerland

Fineman, Kenneth, Fovia Inc., United States

Fleurkens, Hans, FEI Company, Netherlands

Fredrickson, Guy L., United States

Fuentes, Antonio F., Cinvestav Unidad Saltillo, Mexico

Ganeshan, Swetha, Baker Hughes, United States

Gee, Mark, NPL, Great Britain

Gholinia, Ali, University of Manchester, Great Britain

Grandhi, Ramana V., Wright State University, United States

Green, Daniel E., University of Windsor, Canada

Grummon, David S., Michigan State University, United States

GVK, Srikanth Sai, Heraeus Materials Singapore Pte. Ltd., Singapore

Harder, Ross J., Argonne National Laboratory, United States

Henke, Daniel M., United States

Herzing, Andrew A., National Institute of Standards and Technology, United States

Ibarretxe, Julen, University of Basque Country, Spain

Jafari, Meisam, Carnegie Mellon University, United States

Jang, Jae-il, Hanyang University, Korea South

Janz, Eric, Chemineer, United States

Kamal, Kafeel, Canada 
Khan, Athar Said, PSFCL Pakistan Steel Mills, Oman

Kim, Woo Sik, LS-Nikko Copper, Korea South

King, Andrew, ESRF, France

Kutbay, Cihat, American Ceramic Society, United States

LaFollette, James, GeoCorp Inc., United States

Langer, Stephen, National Institute of Standards and Technology, United States

Laundal, Karl Magnus, Teknova AS, Norway

Lawson, James R., Nalco, United States

Lazar, Emanuel A., Institute for Advanced Study, United States

Lhuissier, Pierre, SIMaP - CNRS Grenoble University, France

Lian, Kuo Lung, Taiwan

Llorens, Manel G., Weimac Europa Ltd., Spain

Mason, Jeremy, Lawrence Livermore National Laboratory, United States

Merkle, Arno, Xradia, United States

Midgley, Paul A., University of Cambridge, Great Britain

Momeni, Amir, Hamedan University of Technology, Iran

Morgan, Jason, Freeport McMoran Copper \& Gold, United States

Nekuda Malik, Jennifer A., United States

Ng, Tse Nga, Palo Alto Research Center, United States

Paciornik, Sidnei, DEMa PUC-Rio, Brazil

Palmer, David E., United States

Pang, Hon T., University of Cambridge, Great Britain
Paramguru, Raja Kishore,

Bhubaneswar Institute of Industrial

Technology, India

Parvanian, Amir M., Iran

Podany, Pavel, COMTES FHT A S, Czech Republic

Quinones, David, NORDAM Group, United States

Rano, Alfi, University of Sultan Ageng Tirtayasa, Indonesia

Safriet, Sirina, United States

Schmidt, Soren, Technical University of Denmark, Denmark

Shah, Megna N., UES Inc., United States

Shamamian, Vasgen A., Dow Corning Corporation, United States

Shevock, Bradley T., Virginia Polytechnic Institute \& State University, United States

Shuto, Hiroshi, Nippon Steel, Japan

Sinclair, Ian, University of Southampton, Great Britain

Singh, Chandra Veer, University of Toronto, Canada

Sitzman, Scott D., Oxford Instruments, United States

Song, Jun, McGill University, Canada

Stansbury, Kevin L., Jacobs, United States

Steigerwald, Andrew, United States

Stockinger, Martin, Bohler Schmiedetechnik GmbH \& Co. KG, Austria

Sumi, Toshinari, Kohsei Co. Ltd., Japan

Sundararaghavan, Veera, University of Michigan, United States

Sydnor, James W., United States
Tayon, Wesley A., NASA-LARC, United States

Thierry, Auger, CNRS/ECP, France

Thomas, Arnie, A Thomas Group, United States

Tripathi, Nagendra, Xstrata Nickel, Canada

Tripathy, Prabhat K., Battelle Energy Alliance (Idaho National Laboratory), United States

Tu, Wen J., Exponet Inc., United States

Turner, Todd J., Air Force Research Laboratory, United States

Umantsev, Alexander R., Fayetteville State University, United States

Umasankar, V., VIT University, India

Vakanas, George, IMEC Intel Assignee, Belgium

Van Boxel, Steven, University of Manchester, Great Britain

Wang, Guofeng, University of Pittsburgh, United States

Wang, Song, University of South Carolina, United States

Wang, Yan, Worcester Polytechnic Institute, United States

Wei, Yajun, L 3 Communications Cincinnati Electronics, United States

Wentworth, Adam J., University of Connecticut, United States

West, Geoff, Loughborough University, Great Britain

Yamamoto, Satoshi, Kohsei Co. Ltd., Japan

Yang, Xingbo, Kohsei Co. Ltd., Japan

Yerdon, Elizabeth, Wyman-Gordon, United States

Zhang, Yubin, Denmark 